\title{
BMJ Open Online peer support interventions for chronic conditions: a scoping review protocol
}

\author{
Sarah Elizabeth Patricia Munce, ${ }^{1}$ John Shepherd, ${ }^{2}$ Laure Perrier, ${ }^{3}$ Sonya Allin, ${ }^{4}$ \\ Shane N Sweet, ${ }^{5}$ Jennifer R Tomasone, ${ }^{6}$ Michelle L A Nelson, ${ }^{7}$ Sara J T Guilcher, ${ }^{8}$ \\ Saima Hossain, ${ }^{3}$ Susan Jaglal $1^{1,2,4,9}$
}

To cite: Munce SEP, Shepherd J, Perrier L, et al. Online peer support interventions for chronic conditions: a scoping review protocol. BMJ Open 2017;7:e017999. doi:10.1136/ bmjopen-2017-017999

- Prepublication history and additional material for this paper are available online. To view please visit the journal online (http://dx.doi.org/10.1136/ bmjopen-2017-017999).

Received 5 June 2017 Revised 24 August 2017 Accepted 31 August 2017

\section{CrossMark}

For numbered affiliations see end of article.

Correspondence to Dr Sarah Elizabeth

Patricia Munce;

sarah.munce@mail.utoronto.ca

\section{ABSTRACT}

Introduction Peer support is receiving increasing attention as both an effective and cost-effective intervention method to support the self-management of chronic health conditions. Given that an increasing proportion of Canadians have internet access and the increasing implementation of web-based interventions, online peer support interventions are a promising option to address the burden of chronic diseases. Thus, the specific research question of this scoping review is the following: What is known from the existing literature about the key characteristics of online peer support interventions for adults with chronic conditions?

Methods and analysis We will use the methodological frameworks used by Arksey and 0'Malley as well as Levac and colleagues for the current scoping review. To be eligible for inclusion, studies must report on adults ( $\geq 18$ years of age) with one of the Public Health Agency of Canada chronic conditions or HIV/AIDS. We will limit our review to peer support interventions delivered through online formats. All study designs will be included. Only studies published from 2012 onwards will be included to ensure relevance to the current healthcare context and feasibility. Furthermore, only English language studies will be included. Studies will be identified by searching a variety of databases. Two reviewers will independently screen the titles and abstracts identified by the literature search for inclusion (ie, level 1 screening), the full text articles (ie, level 2 screening) and then perform data abstraction. Abstracted data will include study characteristics, participant population, key characteristics of the intervention and outcomes collected.

Dissemination This review will identify the key features of online peer support interventions and could assist in the future development of other online peer support programmes so that effective and sustainable programmes can be developed.

\section{INTRODUCTION}

More than 20\% Canadian adults live with one of the following chronic diseases: cardiovascular diseases, cancer, chronic respiratory diseases or diabetes. ${ }^{1}$ Since 2000 , the prevalence rates for cardiovascular diseases and chronic respiratory diseases have increased. ${ }^{1}$

\section{Strengths and limitations of this study}

- This review is guided by known methodological frameworks.

- All phases of the review will be conducted in duplicate.

- This review will include the Public Health Agency of Canada definition/list of chronic conditions.

- This review will not include mental health conditions and a variety of other disabilities.

- This review will be limited to English language studies only.

Treatment of chronic diseases consumes $67 \%$ of all direct healthcare costs, and cost the Canadian economy $\$ 190$ billion annually, with $\$ 68$ billion related to treatment costs and the remaining costs related to lost productivity. $^{2}$ In the USA, approximately $25 \%$ of the population has multiple chronic conditions; ${ }^{3}$ among Americans aged 65 years and older, approximately three in four have multiple chronic conditions. ${ }^{4}$ In Australia, more than 7 million people have at least one chronic condition. ${ }^{5}$ Thus, it is imperative to develop and implement effective interventions to manage these chronic conditions.

As a result of this increasing burden, particularly in health services and related costs, individuals with chronic conditions need assistance in learning and maintaining selfcare behaviours that support healthy living, referred to as 'chronic disease self-management'. ${ }^{6-8}$ Chronic condition self-management refers to a person's ability to manage the symptoms, treatment, and physical, psychosocial and lifestyle changes that are associated with living with a chronic condition. ${ }^{7} 8 \mathrm{~A}$ promising intervention is peer support which is receiving increasing attention as both an effective and cost-effective intervention method to support the self-management of chronic health conditions. ${ }^{6}$ 
There is some emerging evidence demonstrating the effectiveness of peer support for individuals with chronic conditions to self-manage their conditions. ${ }^{7-10}$ Peer support in the context of chronic disease management refers to 'support for a person with a chronic condition from someone with the same condition or similar circumstances'. ${ }^{611}$ Individuals who provide peer support offer three types of support based on experiential knowledge: emotional, informational and appraisal. ${ }^{12}$ Emotional support involves caring, empathy and encouragement; informational support involves advice, suggestions with alternative actions and factual feedback relevant to a particular topic; and appraisal support involves affirming feelings, thoughts and behaviours and thus is motivational, encouraging the individual to continue with problem-solving attempts despite setbacks. ${ }^{12}$

There are seven predominant types of peer support models in chronic condition management: professional-led groups that encourage peer interactions, structured peer-led self-management training, peer coaches, community health workers, support groups, telephone-based peer support, and web-based and email-based programmes. ${ }^{6}$ At the same time, it is also important to recognise that there are varied models of peer support, and that treating them as if they are fully defined and highly distinct may lead to overlooking important commonalities. ${ }^{13}$ The current review will focus on peer coaches and web-based and email-based programmes. Given that the majority of households have the internet (eg, 80\% in Canada), ${ }^{14}$ and the increasing use of web-based interventions, there is an increasing need to determine the characteristics of online peer support interventions. ${ }^{611}$ The specific research question of this scoping review is the following: What is known from the existing literature about the key characteristics (eg, duration, frequency, delivery setting, type of intervention, type of support provided-emotional, informational and appraisal, underlying theories for the intervention/ behaviour change techniques/working mechanisms, context etc) of online peer support interventions for adults with chronic conditions?

\section{METHODS AND ANALYSIS}

We will use the methodological frameworks proposed by Arksey and $\mathrm{O}^{\prime}$ Malley ${ }^{15}$ as well as Levac and colleagues ${ }^{16}$ for the current scoping review. These frameworks outline six different stages involved in a scoping review: (1) identifying the research question; (2) identifying relevant studies; (3) selecting studies; (4) charting the data; (5) collating, summarising and reporting the results; and, (6) consulting with relevant stakeholders. ${ }^{15} 16$ The research team has expertise in peer support across chronic conditions (SEPM, JS, SA, SNS, MLAN, SJTG, SJ), online interventions (SA, SEPM, SJ) and knowledge synthesis methods (SEPM, LP, JRT, MLAN, SJTG, SJ). Although traditionally applied to systematic review protocols, the Preferred Reporting Items for Systematic Reviews and Meta-analysis for Protocols ${ }^{17}$ was used to draft this protocol.

\section{Eligibility criteria}

For the purpose of this review, we will include chronic diseases identified by the Public Health Agency of Canada (PHAC), including cancer, heart disease (cardiovascular disease), hypertension, stroke, chronic respiratory diseases (asthma, chronic obstructive pulmonary disease, sleep apnoea), diabetes, inflammatory bowel diseases (Crohn's disease, ulcerative colitis) multiple sclerosis, neurological conditions (eg, Alzheimer's disease and other dementias, cerebral palsy, epilepsy, multiple sclerosis, Parkinson's disease/parkinsonism, traumatic brain injury and traumatic spinal cord injury (SCI)), arthritis and osteoporosis. ${ }^{18}$ Mental illness was excluded from the list given that peer support interventions for this group may have particularly unique features not generalisable to other patient populations with chronic disease. Similarly, in a systematic review on the effectiveness of quality improvement strategies (ie, including patient education and promotion of self-management) for coordination of care to reduce use of healthcare services, Tricco and colleagues ${ }^{19}$ determined that these quality improvement strategies reduced hospital admissions among patients with chronic conditions other than mental illness, indicating that different approaches are needed for mental health. To this list, we have also included HIV/AIDS (which, from our preliminary research, has a significant amount of literature on peer support interventions, ${ }^{20}$ and is increasingly being viewed as a chronic condition). Studies including individuals with comorbidities (including mental illness) will be accepted. Thus, to be eligible for inclusion, the studies must report on adults ( $\geq 18$ years of age) with one of these PHAC chronic conditions (excluding mental illness) or HIV/AIDS. We will limit our review to peer support interventions delivered through online formats. Peer support in the context of chronic disease management is operationalised as 'support for a person with a chronic condition from someone with the same condition or similar circumstances'. ${ }^{11}$ This type of support could be emotional, informational and/or appraisal. ${ }^{12}$ Examples of online peer interventions could include Skypebased discussions, social media peer interactions or text messages from a peer(s). Therefore, interventions that are professional-led groups that encourage face-to-face peer interactions, involve community health workers who are not peers (ie, healthcare professionals), support groups and provide telephone-based peer support will be excluded. All study designs will be included (eg, observational studies, randomised controlled trials and qualitative studies). Only studies published from January 2012 to April 2017 will be included. We have included studies from the last 5 years to ensure relevance to the current healthcare context, in technology and feasibility. Furthermore, only English language studies will be included, which may result in a predisposition in results towards English language speaking countries. 


\section{Search strategy and information sources}

Literature search strategies will be developed using medical subject headings and text words related to chronic conditions and peer support interventions. Studies will be identified by searching Medline (OVID interface), CINAHL (EBSCO interface), EMBASE (OVID interface), PsycInfo (OVID interface), and Cochrane Central Register Controlled Trials (Cochrane Library) and PEDro (physiotherapy evidence database). The search strategy for Medline can be found in the online supplementary file 1. A hand search of the reference lists from reviews and selected articles will be made to ensure literature saturation. Finally, experts in the field of peer support will be contacted and consulted in order to ensure that all relevant data are obtained, including members of the research team. An information specialist (LP) who is expert in systematic and scoping reviews will conduct all of the literature searches.

\section{Study selection}

To promote the reliability of screening by the two reviewers, a pilot test of the level 1 screening form based on the criteria outlined above will be conducted on a random sample of approximately 100 articles. The $\kappa$ statistic will then be calculated to determine the interrater reliability for study inclusion. ${ }^{21}$ If low agreement is observed, the inclusion and exclusion criteria will be simplified to increase the consistent application of the selection criteria. Two reviewers will independently screen the titles and abstracts identified by the literature search for inclusion using the screening form (ie, level 1 screening). The full text of potentially relevant articles will then be collected and screened to determine final inclusion (ie, level 2 screening). A pilot test of the level 2 screening form will be performed on approximately $1 \%$ of the articles and the inter-rater reliability for study inclusion will also be calculated. ${ }^{21} \mathrm{~A}$ third reviewer who is knowledgeable in the research area will be available to resolve conflicts, if necessary.

\section{Data items and data collection process}

Abstracted data will include study characteristics (eg, year of publication, country of study), participant population (eg, chronic disease condition, income, ethnicity, age, sex, education), key characteristics of the intervention (eg, duration, frequency, delivery setting, type of intervention, type of support provided-emotional, informational and appraisal, underlying theories for the intervention/ behaviour change techniques/working mechanisms, context, etc) and type of outcome collected (if applicable) as well as results (ie, efficacy or effectiveness). Outcomes reported may include, but are not limited to, self-efficacy, depression and quality of life. In fact, we aim to identify the variety of reported impacts. Additional categories may be identified through the completion of the search and through discussions with the research team and key stakeholders. A data abstraction form will also be pilot tested and modified if poor agreement is observed. For example, any wording on the form that may be related to poor agreement will be reviewed and improved. Two reviewers will independently abstract all of the data and a discussion or the involvement of a third reviewer will resolve disagreements. Study quality will not be evaluated as the purpose of a scoping review is to identify gaps in the literature and future areas for a systematic review. ${ }^{1516}$ DistillerSR will be used to manage the records and data throughout the review.

\section{Synthesis}

The data from this scoping review will be summarised quantitatively using numerical counts and qualitatively using thematic analysis and will be grouped by chronic condition type (eg, what kind of underlying theories are found in online peer support interventions in cancer?). These data will be coded/analysed manually. The results of this review will determine the key characteristics (eg, duration, frequency, delivery setting, type of intervention, type of support provided-emotional, informational and appraisal, underlying theories for the intervention/ behaviour change techniques/working mechanisms, context, etc) of online peer support interventions for adults with chronic conditions. This scoping review will identify gaps in the literature as well as future areas for study either via implementation studies, consensus meeting or systematic review.

\section{DISSEMINATION}

Knowledge translation activities will occur at the beginning of the review and continue throughout with dissemination of the research question to key stakeholders such as the Ontario Ministry of Health and Long-term Care, the Ontario Peer Development Initiative, March of Dimes Canada, SCI Canada, Rick Hansen Institute and the Ontario Neurotrauma Foundation. End-of-grant knowledge translation could also take place through these organisations and their outlets (eg, print and online newsletters) as well as through traditional knowledge translation mechanisms (eg, peer-reviewed journals and conference). For example, the results of the scoping review will be presented at meetings locally, nationally and internationally (eg, National Conference on Peer Support, American Medical Informatics Association) and published in a peer-reviewed journal so that results are available to the appropriate academic and clinical audiences. Finally, partnerships with local clinical programmes and/or research initiatives will be made so that the results are disseminated in a timely and effective manner.

There will be expected limitations and strengths to this review. For example, this review will not include (primary) mental health conditions and a variety of other disabilities. A number of problem-solving approaches for mental health conditions are emerging as quite effective when administered by non-professionals, including in low-resource settings. ${ }^{22-24}$ The possibility of implementing these online would represent exciting advances 
in the field of peer support, and thus, a future, separate systematic/scoping review on online peer support interventions for mental health conditions should be considered. Furthermore, this review will be limited to English language studies only. Lastly, our review will be limited to the published research literature. We acknowledge that we will be excluding reports on available, relevant programmes but not published in these arenas. However, the currently proposed scoping review has a number of strengths-it is guided by known methodological frameworks and all phases will be conducted in duplicate. This review will contribute to critical and emerging perspectives on peer support. For example, the results of the review will identify the key features of online peer support interventions, and in doing so, assist in the future development of other online peer support programmes (ie, so that effective and sustainable programmes can be developed). Similarly, the current review will provide considerations for programmes that are not yet in the online format so that they can be modified with features that are unique to the online environment.

\section{Author affiliations}

${ }^{1}$ Toronto Rehabilitation Institute, University Health Network, Toronto, Ontario, Canada ${ }^{2}$ Rehabilitation Sciences Institute, University of Toronto, Toronto, Ontario, Canada ${ }^{3}$ Gerstein Science Information Centre, University of Toronto, Toronto, Ontario, Canada

${ }^{4}$ Department of Physical Therapy, University of Toronto, Toronto, Ontario, Canada ${ }^{5}$ Department of Kinesiology and Physical Education,Center for Interdisciplinary Research in Rehabilitation of Greater Montreal, McGill University, Montreal, Quebec, Canada

${ }^{6}$ School of Kinesiology and Health Studies, Queen's University, Kingston, Ontario, Canada

${ }^{7}$ Department of Bridgepoint Collaboratory, Lunenfeld-Tanenbaum Research Institute, Toronto, Ontario, Canada

${ }^{8}$ Leslie Dan Faculty of Pharmacy, University of Toronto, Toronto, Ontario, Canada

${ }^{9}$ Institute of Health Policy, Management, and Evaluation, University of Toronto, Toronto, Ontario, Canada

Contributors SEPM conceived of the scoping review, together with JS, LP and SJ. SEPM wrote the first draft of the protocol and is the guarantor of the review. SEPM, $\mathrm{JS}$ and LP were involved in the preliminary literature review. LP conducted the literature search and provided methodological expertise (knowledge synthesis). JS, SA, SNS, JRT, SJTG and MLAN provided critical content expertise on peer support that was integrated into the current protocol. SEPM, JS, LP, SA, SNS, JRT, MLAN, SJTG, SH and SJ were involved in editing and revising the protocol for important intellectual content, and approved the final version of the protocol.

Funding This work was supported by the Ontario Neurotrauma Foundation (ONF) and the Rick Hansen Institute (RHI). The ONF and RHI had no role in developing this protocol. SEPM is/has been supported by a Heart and Stroke Foundation of Canada Focus on Stroke Fellowship, Toronto Rehabilitation Institute-University Health Network, Canadian Institutes of Health Research Fellowship, and a European Stroke Research Foundation Investigator Award. SNS is supported by a salary award from FRQS Chercheurs-Boursiers, Junior 1 program.

Competing interests None declared.

Patient consent Detail has been removed from these case descriptions to ensure anonymity. The editors and reviewers have seen the detailed information available and are satisfied that the information backs up the case the authors are making.

Provenance and peer review Not commissioned; externally peer reviewed.

Open Access This is an Open Access article distributed in accordance with the Creative Commons Attribution Non Commercial (CC BY-NC 4.0) license, which permits others to distribute, remix, adapt, build upon this work non-commercially, and license their derivative works on different terms, provided the original work is properly cited and the use is non-commercial. See: http://creativecommons.org/ licenses/by-nc/4.0/

(c) Article author(s) (or their employer(s) unless otherwise stated in the text of the article) 2017. All rights reserved. No commercial use is permitted unless otherwise expressly granted.

\section{REFERENCES}

1. Public Health Agency of Canada. How healthy are Canadians? A trend analysis of the health of Canadians from a healthy living and chronic disease perspective. Ottawa, ON: Public Health Agency of Canada, 2016.

2. Elmslie K. Against the growing burden of disease. Ottawa, ON: Public Health Agency of Canada, 2016.

3. US Department of Health and Human Services. Multiple chronic conditions - a strategic framework: optimum health and quality of life for individuals with multiple chronic conditions. Washington, DC: US Department of Health and Human Services, 2010.

4. Gerteis J, Izrael D, Deitz D, et al. Multiple chronic conditions chartbook. Rockville, MD: Agency for Healthcare Research and Quality, 2014.

5. Australian Institute of Health and Welfare. Authoritative information and statistics to promote better health and well-being: chronic diseases. http://www.aihw.gov.au/chronic-diseases.

6. Heisler M. Overview of peer support models to improve diabetes self-management and clinical outcomes. Diabetes Spectrum 2007;20:214-21.

7. de Silva D. Helping people help themselves: a review of the evidence considering whether or not it is worthwhile to support selfmanagement. London, UK: The Health Foundation, 2011.

8. Health Council of Canada. Self-management support for Canadians with chronic conditions: a focus for primary care. http://www. healthcouncilcanada.ca/rpt_det.php?id=372.

9. Cook JA, Copeland ME, Corey L, et al. Developing the evidence base for peer-led services: changes among participants following Wellness Recovery Action Planning (WRAP) education in two statewide initiatives. Psychiatr Rehabil J 2010;34:113-20.

10. Chronic Illness Alliance. Peer support for chronic and complex conditions: a literature review. http://www.chronicillness.org.au.

11. Lauckner HM, Hutchinson SL. Peer support for people with chronic conditions in rural areas: a scoping review. Rural Remote Health 2016;16:3601.

12. Dennis CL. Peer support within a health care context: a concept analysis. Int J Nurs Stud 2003;40:321-32.

13. Eng E, Parker E, Harlan C. Lay health advisor intervention strategies: a continuum from natural helping to paraprofessional helping. Health Educ Behav 1997;24:413-7.

14. Statistics Canada. Internet in 4 out of 5 households. Ottawa, ON: Statistics Canada, 2010.

15. Arksey H, O'Malley L. Scoping studies: towards a methodological framework. Int J Soc Res Methodol 2005;8:19-32.

16. Levac D, Colquhoun H, O'Brien KK. Scoping studies: advancing the methodology. Implement Sci 2010;5:69.

17. Shamseer L, Moher D, Clarke M, et al. Preferred reporting items for systematic review and meta-analysis protocols (PRISMA-P) 2015: elaboration and explanation. BMJ 2015;349:g7647.

18. Public Health Agency of Canada. Chronic diseases http://www.phacaspc.gc.ca/cd-mc/index-eng.php.

19. Tricco AC, Antony J, Ivers NM, et al. Effectiveness of quality improvement strategies for coordination of care to reduce use of health care services: a systematic review and meta-analysis. CMAJ 2014;186:E568-78.

20. Prestage G, Brown G, Allan B, et al. Impact of peer support on behavior change among newly diagnosed Australian gay men. $J$ Acquir Immune Defic Syndr 2016;72:1-71.

21. Landis JR, Koch GG. The measurement of observer agreement for categorical data. Biometrics 1977;33:159-74.

22. Rahman A, Malik A, Sikander S, et al. Cognitive behaviour therapybased intervention by community health workers for mothers with depression and their infants in rural Pakistan: a cluster-randomised controlled trial. Lancet 2008;372:902-9.

23. Chowdhary N, Anand A, Dimidjian S, et al. The healthy activity program lay counsellor delivered treatment for severe depression in India: systematic development and randomised evaluation. Br J Psychiatry 2016;208:381-8.

24. Rahman A, Hamdani SU, Awan NR, et al. Effect of a multicomponent behavioral intervention in adults impaired by psychological distress in a conflict-affected area of Pakistan: a randomized clinical trial. JAMA 2016;316:2609-17. 